\title{
Detection of hepatocyte growth factor/scatter factor receptor (c-Met) in axillary drainage after operations for breast cancer using reverse transcriptase-polymerase chain reaction
}

\author{
Ron Greenberg ${ }^{1}$, Ignat Schwartz ${ }^{2}$, Yehuda Skornick ${ }^{1}$ and Ofer Kaplan ${ }^{1}$ \\ ${ }^{1}$ Department of Surgery A, Tel-Aviv Medical Center, Tel-Aviv University, Tel-Aviv, Israel \\ ${ }^{2}$ Department of Pathology and Cancer Research Center, Tel-Aviv Medical Center, Tel-Aviv University, Tel-Aviv, Israel \\ Corresponding author: Ron Greenberg (e-mail: rongree@mailcity.com)
}

Received: 21 Oct 2002 Revisions requested: 6 Dec 2002 Revisions received: 9 Jan 2003 Accepted: 4 Feb 2003 Published: 6 Mar 2003

Breast Cancer Res 2003, 5:R71-R76 (DOI 10.1186/bcr588)

(C) 2003 Greenberg et al., licensee BioMed Central Ltd (Print ISSN 1465-5411; Online ISSN 1465-542X). This is an Open Access article: verbatim copying and redistribution of this article are permitted in all media for any purpose, provided this notice is preserved along with the article's original URL.

\begin{abstract}
Background: The diverse biological effects of hepatocyte growth factor/scatter factor (HGF/SF) are mediated by c-Met, which is preferentially expressed on epithelial cells. Met signaling has a role in normal cellular activities, and may be associated with the development and progression of malignant processes. In this study we examined whether Met can be detected in the axillary drainage from patients who underwent conservative operations for breast cancer, and its prognostic significance.

Methods: Thirty-one consecutive patients with invasive ductal carcinoma of the breast suitable for breast-conserving treatment were studied. The output of the drain that had been placed in the axilla during the operation was collected, and the presence of Met and $\beta$-actin were assessed by reverse transcriptase-polymerase chain reaction (RT-PCR) assays. The data were compared with the pathological features of the tumor and the axillary lymph nodes, and with the estrogen receptor and progesterone receptor status.
\end{abstract}

Results: RT-PCR of the axillary lymphatic drainage was positive for Met in $23(74.2 \%)$ of the patients. Positive assays were correlated with increasing tumor size and grade, with capillary and lymphatic invasion, and with lymph node metastasis ( $P<0.02$, for all comparisons). All 12 patients with axillary lymph node metastases had positive assays for Met, compared with $57.9 \%$ of patients without lymph node metastases. All five patients with tumor involvement in the margins of the resection had positive assays for Met in their lymphatic fluid, compared with 18 of 26 positive assays $(69.2 \%)$ for patients without involved margins $(P<0.04)$. Finally, Met showed negative correlations with positivity for estrogen receptor and progesterone receptor $(P<0.02)$.

Conclusion: Met can be detected in the axillary fluids of patients with breast cancer and its expression in the axillary drainage may have potential as a prognostic factor. This finding might be relevant to therapeutic considerations, because a positive assay for Met in histologically node-negative patients might point to the need to search for node microinvasion or involvement of the excision margins with tumor.

Keywords: axillary fluid, breast cancer, hepatocyte growth factor, Met, prognosis

\section{Introduction}

As many as $30 \%$ of patients with breast cancer who have undergone curative surgery and show no evidence of locoregional or distant disease still have recurrent disease over $5-10$ years $[1,2]$. Some of these treatment failures may be attributed to residual disease in the breast or axillary lymph nodes [3]. The limitation of routine histopathologic examination of the tumor margins and the dissected lymph node specimen is well known [4]. Contemporary methods of detection, including computed tomography, magnetic resonance imaging, bone scintigraphy and flow cytometry, all have limited sensitivity and specificity $[5,6]$. Micrometastases can be found by immunohistochemistry or polymerase chain reaction in $10-30 \%$ of the patients previously deemed free of disease by conventional histological methods $[7,8]$. The prognostic importance of

$\mathrm{ER}=$ estrogen receptor; HGFA = hepatocyte growth factor activator; HGF/SF = hepatocyte growth factor/scatter factor; PR = progesterone receptor; $\mathrm{RT}-\mathrm{PCR}=$ reverse transcriptase-polymerase chain reaction. 
micrometastases found with these sensitive methods are now being evaluated [9-11].

Hepatocyte growth factor/scatter factor (HGF/SF) is a paracrine factor produced primarily by mesenchymal cells. HGF/SF induces mitogenic and morphogenic changes, including rapid membrane ruffling, formation of microspikes, and increased cellular motility $[12,13]$. The diverse biological effects of HGF/SF are all mediated by Met, which is preferentially expressed on epithelial cells [14]. In vivo this receptor-ligand pair is essential for normal embryonic development $[15,16]$. Whereas Met signaling clearly has a role in normal cellular processes, this signaling pathway has also been implicated in tumor development and progression. Met signaling can increase tumorigenicity, induce cell motility, and enhance invasiveness in vitro and metastasis in vivo [14,17-20]. In addition, Met signaling can increase the production of protease and urokinase, which are associated with extracellular matrix/basal membrane degradation and are important for metastasis $[14,19]$.

Operations for breast cancer include either mastectomy or breast-conserving surgery, consisting of wide local excision of the tumor with margins of intact breast tissue ('lumpectomy') and axillary lymph node dissection. Drains are inserted in the dissected axilla in most of these operations, to avoid the accumulation of lymphatic fluid. The goals of this study were to examine whether Met can be detected by reverse transcriptase-polymerase chain reaction (RT-PCR) in the axillary drainage from patients who have undergone conservative operations for breast cancer, and to assess the correlations between the mRNA expression of Met in the collected fluid and prognostic factors of breast cancer.

\section{Materials and methods Patients and operations}

Thirty-one consecutive women with invasive ductal carcinoma of the breast, who were suitable for breast-conserving treatment, were studied. We included only patients who underwent conserving breast surgery so that we could evaluate the correlations of Met-HGF/SF expression with both the tumor margins and the status of the axillary lymph nodes. The diagnosis of cancer was established by needle core biopsy ('Trucut'), which was performed 2 weeks before the operation. All patients underwent wide local excision and axillary lymph node dissection by the same team. Non-palpable tumors were localized by mammography before surgery by wire insertion. During operations a wide resection around the wire was performed, followed by mammographic confirmation of complete resection of the tumors. The axillary dissection was performed with a separate incision, and level I and II axillary lymph nodes were removed. The breast incisions postoperatively by closed vacuum drains (ch-14; Biometrix). The output of the drain was collected and measured every 24 hours; the drains were removed when the output was less than $25 \mathrm{ml}$ per $24 \mathrm{~h}$. The presence of Met-HGF/SF and $\beta$-actin were assessed in the fluid, which was collected during the second postoperative day because during the first 24 hours it might contain many erythrocytes and debris.

\section{Pathological examinations}

The resected specimen was covered circumferentially by black Indian ink and Bouins solution and then was sliced into $5 \mathrm{~mm}$ slices. Each slice was evaluated macroscopically for the presence of tumor and its distance from the margins of the specimen. All slices involved with tumor were paraffin embedded, sliced again into $4 \mu \mathrm{m}$ slides, and stained with hematoxylin-eosin. Microscopical evaluation was performed by one pathologist for margin involvement, tumor type, size, grade, capillary or lymphatic invasion, and the distance from the margins. All axillary lymph nodes were paraffin embedded, sliced into $4 \mu \mathrm{m}$ slides and assessed for the presence of micrometastases.

\section{Receptor assays}

Estrogen receptor (ER) and progesterone receptor (PR) were assessed in the tumor by immunohistochemical assay with mouse monoclonal antibodies (NCL-ER-6F11 and NCL-PGR-312) in accordance with the manufacturer's instruction (Novocastra Laboratories). We used the 'quick score', a simple combination of the proportion of cells staining plus a measure of intensity of staining [21]. A cut-off value of 2 or more was taken as negative for ER or PR.

\section{RT-PCR assays}

Total RNA was extracted from axillary lymphatic fluid with the Tri Reagent procedure, in accordance with the manufacturer's instruction (Sigma). Reverse transcription was performed with $1-2 \mu \mathrm{g}$ of total RNA. The first strand of cDNA was generated with $0.5 \mu \mathrm{g}$ of $(\mathrm{dT})_{15}$ primer (Gibco-BRL) using 200 units of subscripts II RNAse$\mathrm{H}$-reverse transcriptase (Gibco-BRL). This was incubated for $50 \mathrm{~min}$ at $42^{\circ} \mathrm{C}$, followed by inactivation for $15 \mathrm{~min}$ at $70^{\circ} \mathrm{C}$. To detect Met transcript, PCR was performed on $3 \mu \mathrm{l}$ of CDNA with MP1 primer (5'GGAATCGAGCTGCGAGA-3') and MP2 primer (5'TCCAACATGCAGTTTCTTGC-3'). To detect $\beta$-actin transcript, PCR was performed on $3 \mu \mathrm{l}$ of $\mathrm{cDNA}$ with MP1 primer (5'-CTCTTCCAGCCTTCCTTCCT-3') and MP2 primer (5'-AGCACTGTGTTGGCGTACAG-3'). Cycling conditions consisted of 35 cycles with denaturation steps at $94^{\circ} \mathrm{C}$ for $30 \mathrm{~s}$, hybridization steps at $55^{\circ} \mathrm{C}$ for $30 \mathrm{~s}$ and an extension step at $72^{\circ} \mathrm{C}$ for $1 \mathrm{~min}$. The $\beta$-actin and c-Met RT-PCRs were performed simultaneously, under the same conditions. The limit of sensitivity of the RT-PCR system for Met was $1 \mathrm{pg}$ of total RNA. 


\section{Immunohistochemical analyses}

Staining was performed with an antibody against hepatocyte growth factor- $\alpha$ receptor (HGF $\alpha-\mathrm{R}$; $\mathrm{H}-145$; Santa Cruz Biotechnology, Santa Cruz, California, USA). Sections $(5 \mu \mathrm{m})$ mounted on Super Frost/plus glass (Menzel and Claser, both in Braunschweig, Germany), were processed by a labelled streptavidin-biotin method with a Histostain Plus kit (Zymed, San Francisco, California, USA). Heat-induced antigen retrieval was performed by temperature-controlled microwave treatment with an H2800 model processor (Energy Beam Sciences Inc, Agawan, Massachusetts, USA) for $12 \mathrm{~min}$ in $10 \mathrm{mM}$ citrate buffer, $\mathrm{pH} 6.0$, at $97^{\circ} \mathrm{C}$. The sections were treated for 5 min with $3 \% \mathrm{H}_{2} \mathrm{O}_{2}$, followed by a 10 min incubation with the universal blocker, Cas-block (Zymed). The sections were incubated for $32 \mathrm{~min}$ with 1:20 diluted HGF $\alpha-R$ antibody. A biotinylated second antibody was applied for $10 \mathrm{~min}$, followed by incubation with horseradish peroxidase-conjugated streptavidin for $10 \mathrm{~min}$. The slides were washed with Optimax wash buffer (BioGenex, San Ramon, California, USA) after each incubation. The immunoreaction was revealed by a horseradish peroxidase-based chromogen. The sections were then counterstained and cleared in xylene. Controlled staining was performed with human liver tissue. The presence of brown (diaminobenzidine) staining of the cell membranes of tumor cells was interpreted as positive reactivity.

\section{Control group}

To verify that in normal persons there is no Met in the axilla we examined the axillary drainage in 20 patients with malignant melanoma who were found to have negative axillary sentinel lymph node. The lesions were located in the upper limbs, and the lymph node basins were found to be in the axilla. Sentinel lymph nodes were found in all these patients and none was involved with melanoma in frozen sections or in paraffin-embedded and stained slices.

\section{Statistical evaluation}

Correlations between the various clinicopathological parameters and RT-PCR assays in lymphatic fluid were analyzed by the $\chi^{2}$ test $(P<0.05)$.

\section{Results}

Thirty-one consecutive female patients who underwent breast-conserving surgery for operable invasive duct carcinoma of the breast between 1 January and 30 June 2000 were included. Patients with tumors that invaded the chest wall or skin, or with inflammatory carcinoma, were excluded. The mean age was $58 \pm 16$ years. Twenty-three patients (74.2\%) underwent lumpectomy for palpable masses, and in eight women (25.8\%) wireguided excision of non-palpable tumors was performed. The tumor size was $0-1 \mathrm{~cm}$ in 10 women (32.2\%), $1-2 \mathrm{~cm}$ in 9 (29.0\%), 2-5 cm in 11 (35.4\%), and larger

\section{Figure 1}

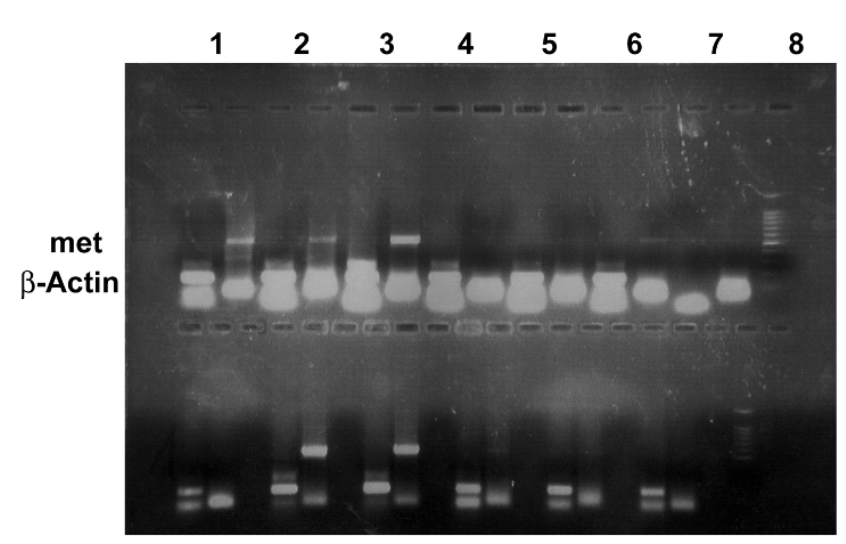

Reverse transcriptase-polymerase chain reaction (RT-PCR) amplification of Met and $\beta$-actin mRNA obtained from lymphatic fluids from women with breast cancer. Met and $\beta$-actin expression were examined by RT-PCR, and the results of agarose-gel electrophoresis of some of the patients are shown.

than $5 \mathrm{~cm}$ in 1 (3.2\%). Four patients (12.9\%) had grade I tumors, $19(61.3 \%)$ had grade II lesions, and grade III tumors were found in 8 (25.8\%). Lymphatic and capillary invasion were noted in 10 (32.2\%) and 11 (35.4\%) patients, respectively. While attempting to achieve free margins, five patients $(16.1 \%)$ had to undergo re-excision owing to incomplete resections. Two of these patients had wire-guided excisions.

The collected axillary fluids were assessed by RT-PCR for Met and $\beta$-actin. The $\beta$-actin RT-PCR served as positive control and gave strong signals in all cases, indicating that both RNA preparation and cDNA synthesis were successful. The RT-PCR assays were positive for Met in 23 $(74.2 \%)$ of the breast cancer patients (Fig. 1). In all of the patients in the control group, RT-PCR gave positive results for $\beta$-actin but was negative for Met.

The correlations between tumor size and the presence of Met in the axillary fluid are shown in Table 1. Positive RT-PCR assays for Met in the lymphatic fluids were associated with increasing tumor size. Eleven of 19 patients (57.9\%) with T1 tumors (tumor size $0-2 \mathrm{~cm}$ ) had positive Met in the axillary fluid $(50 \%$ and $66 \%$ for tumors $0-1$ and $1-2 \mathrm{~cm}$ in size respectively), compared with 12 of 12 patients $(100 \%)$ with T2 and T3 tumors $(P<0.01)$.

The average number $( \pm S D)$ of axillary lymph nodes was $17 \pm 4$, and all dissected nodes were examined for the presence of metastases. Twelve patients (38.7\%) had axillary lymph node metastases as revealed by hematoxylin-eosin staining. The correlation between metastatic axillary lymph node involvement and the presence of Met in the lymphatic fluid is shown in Table 2. All 
Table 1

\begin{tabular}{lc}
\multicolumn{2}{l}{ Correlation between Met status and tumor size } \\
\hline Tumor size $(\mathrm{cm})$ & Met-positive patients (\%) \\
\hline $0-1$ & $5 / 10(50)$ \\
$1-2$ & $6 / 9(66)$ \\
$2-5$ & $11 / 11(100)$ \\
$>5$ & $1 / 1(100)$ \\
\hline
\end{tabular}

12 patients with axillary lymph node metastases had positive RT-PCR assays for Met, compared with $57.9 \%$ (11 of 19) positive assays for women without lymph node metastases $(P<0.01)$.

All five patients with tumor involvement in the margins of the resection had positive RT-PCR assays for Met in their lymphatic fluid, compared with 18 of 26 positive assays $(69.2 \%)$ for patients without involved margins $(P<0.04)$. The associations between tumor grade, capillary and lymphatic invasion, and the presence of Met in the axillary fluid are presented in Table 3. Patients with lymphatic invasion of the tumor were all Met-positive (10 of 10), and patients without lymphatic invasion had $61.9 \%$ (13 of 21 ) positive assays for Met $(P<0.02)$. Capillary invasion of the tumor was also found to be in correlation with Met; although all 11 patients with capillary invasion had positive RT-PCR assays for Met in their axillary fluid, the assays were positive in only 60\% (12 of 20) patients without capillary invasion $(P<0.02)$. The presence of Met in the axillary drainage was also correlated with higher tumor grade: it was found in 50\% of grade I tumors, compared with $87.5 \%$ of grade III lesions $(P<0.05)$.

The correlations between the ER and PR status and RT-PCR assays for Met in the axillary fluid are shown in Table 4. Seventeen patients had ER-negative tumors, and $16(94.1 \%)$ of these had Met-positive assays in the axillary fluid. In seven (87.5\%) of the eight patients with Met-negative assays the ER were positive. Similarly, 21 patients had PR-negative tumors, and 20 (95.2\%) of these were positive for Met. PR-positive tumors were found in seven of eight patients (87.5\%) in whom assays for Met in the axillary drainage were negative.

All the paraffin-embedded sections of the resected primary breast cancers were assessed by immunohistochemistry staining for Met. In 28 patients (90.3\%) the primary tumor stained positively for Met and none of the tumors that had negative staining had a positive Met RT-PCR assay in the axillary drainage. In addition, the patients with negative staining had no involvement of
Table 2

Correlation between Met status and axillary lymph node involvement

\begin{tabular}{lc}
\hline Lymph node involvement & Met-positive patients $(\%)$ \\
\hline None & $11 / 19(57.9)$ \\
$1-3$ & $8 / 8(100)$ \\
$4-10$ & $4 / 4(100)$ \\
\hline
\end{tabular}

Table 3

Correlation between Met status, capillary and lymphatic invasion and tumor grade

\begin{tabular}{lcc}
\hline Measure & Status & Met-positive patients (\%) \\
\hline Capillary invasion & Present & $11 / 11(100)$ \\
& Absent & $12 / 20(60)$ \\
Lymphatic invasion & Present & $10 / 10(100)$ \\
& Absent & $13 / 21(61.9)$ \\
Grade & I & $2 / 4(50)$ \\
& II & $14 / 19(73.6)$ \\
& III & $7 / 8(87.5)$ \\
\hline
\end{tabular}

Table 4

Correlation between Met status, estrogen receptor and progesterone receptor

\begin{tabular}{lc}
\hline Receptor status & Met-positive patients (\%) \\
\hline ER-positive & $7 / 14(50)$ \\
ER-negative & $16 / 17(94.1)$ \\
PR-positive & $3 / 10(30)$ \\
PR-negative & $20 / 21(95.2)$ \\
\hline
\end{tabular}

ER, estrogen receptor; PR, progesterone receptor.

\section{Discussion}

HGF/SF is synthesized as a pro-HGF, and once activated by the HGF activator (HGFA), the complex HGF/SF-Met affects numerous normal cellular processes [22]. HGF/SF-Met is also involved in malignant transformation, presumably as a mediator in interaction between tumor and stroma, which enhances tumor progression, and also angiogenesis [23,34]. In invasive ductal carcinoma, stronger expression of HGF/SF seems to be associated with tubule formation [25]. HGF/SF is overexpressed in breast carcinoma in situ and invasive ductal carcinoma in comparison with normal breast tissue. Normal mammary 
ducts within infiltrating cancer showed intermediate levels of HGF/SF. This finding suggests that the expression of these proteins in breast cancer is regulated by soluble factors produced by the tumor cells $[26,27]$. High levels of expression of HGF and Met are associated with invasive breast cancer, and might be causally linked to early recurrences, metastatic disease and shortened survival of breast cancer patients $[28,29]$. High levels of HGF/SF detected within breast tumor extracts are correlated with larger tumor size and shorter relapse-free and overall survival compared with tumors with low HGF/SF concentration [30].

The activation of HGF/SF by HGFA might be modified by the two HGFA inhibitors, HAl-1 and HAl-2. Highly invasive breast cancer cells express large amounts of HGF and Met, and no HAl-1, whereas breast cancer cells with low invasive potential have low levels of HGF and Met, and high levels of HAl-1 [22]. In a mouse model system HGF antagonists suppressed the conversion of pancreatic tumors from carcinoma in situ into invasive cancer [23]. It seems that regulation of the HGF/SF stimulation and inhibition activities is associated with the metastatic potential of tumor cells, and knowing the status of HGFA, HAl-1 and $\mathrm{HAl}-2$, in addition to Met, might provide useful information.

HGF/SF and Met have been found in a variety of tumors [31-33], and in lymph nodes of patients with no tumor [34], but never in the fluid drained from the tumor bed or the lymph node basin. In this study we evaluated whether Met can be detected in the axillary drainage of breast cancer patients, and the significance of its expression in the lymphatic fluid. Studying the expression of Met in the axillary fluid is a simple, non-invasive procedure because drains are routinely inserted during axillary lymph node dissections. The collected fluid is readily available, and RT-PCR is a routine, short assay with minimum artefacts.

The axillary fluid after breast and axillary lymph node operations includes erythrocytes, lymphocytes, epithelial cells and tumor cells. One of the goals of this work was to examine whether tumor cells can be detected in the axillary drainage by RT-PCR assays for Met. To determine the source of Met in the axillary fluid in breast cancer patients and to exclude the possibility that the source was related to surgical trauma, we evaluated a control group of melanoma patients with negative axillary sentinel lymph nodes. In none of the control patients was the axillary drainage Met-positive. In 28 patients the primary tumor stained positively for Met and none of the tumors that had negative immunostaining had a positive Met RT-PCR assay in the axillary drainage. Thus, it seems likely that the source of Met in the axillary fluid in node-negative breast cancer patients is the tumor in the breast.
The results show that Met can be detected in the axillary drainage, and although the number of patients was not high, Met is associated with unfavorable prognostic factors. Positive assays for Met are correlated with tumor size, grade, lymphatic invasion, tumor involvement of the margins of the resected specimen, the existence of metastases in the lymph nodes, and the numbers of lymph nodes with tumor. Met was associated with larger tumors, and in none of the patients with tumors larger than $2 \mathrm{~cm}$ was the assay negative. Moreover, Met was never negative in patients with metastatic carcinoma in the lymph node. Negative staining for ER and PR is associated with unfavorable prognosis, and the assays for Met in the axillary fluid were positive in most receptor-negative tumors.

One could postulate that because Met was highly correlated with tumor size, its expression implies aggressive behavior of the malignant processes. The significance of HGF/SF as a marker of poor prognosis might also be associated with its effects on acquired resistance to anticancer drugs $[35,36]$. HGF/SF protects cancer cells from DNA-damaging chemotherapy agents through pathways involving signaling from Met to phosphoinositide 3-kinase and c-Akt [29]. Human breast cancer cells, preincubated with HGF/SF and then exposed to Adriamycin, exhibit an altered pattern of gene expression compared with cells treated with Adriamycin alone. Cells treated with HGF/SF and Adriamycin also had modified cell line regulation and signal transduction that might suggest mechanisms by which HGF/SF exerts its protective activity [31].

To our knowledge, this is the first report of the expression of Met in the axillary drainage in patients who have undergone operations for breast cancer. The results suggest that the expression of Met in the axillary drainage might have prognostic significance. More importantly, its expression in histologically node-negative patients point to the need to search for node micrometastasis or involvement of the excision margins by tumor. Therefore, RT-PCR for Met in the axillary fluid of patients who undergo breast-conserving surgery for breast cancer could influence therapy. The results of this study justify prospective investigation on a larger scale, and the clinical significance of Met positivity in the axillary drainage fluid in breast cancer patients should be defined by an extended follow-up study.

\section{Competing interests}

None declared.

\section{References}

1. Berois N, Varangot M, Aizen B, Estrugo R, Zarantonelli L, Fernandez P, Krygler G, Simonet F, Barrios E, Muse I, Osinaga E: Molecular detection of cancer cells in bone marrow and peripheral blood of patients with operable breast cancer. Comparison of CK19, MUC1 and CEA using RT-PCR. Eur J Cancer 2000, 36:717-723.

2. Yeu-Tsu ML: Patterns of metastasis and natural courses of breast carcinoma. Cancer 1994, 74:2403-2413. 
3. Hellman S: Natural history of small breast cancers. J Clin Oncol 1994, 12:2229-2234.

4. International (Ludwig) Breast Cancer Study Group: Prognostic importance of occult axillary lymph node micrometastases from breast cancers. Lancet 1990, 335:1565-1568.

5. Frank JA, Ling A, Patronas NJ: Detection of malignant bone tumors: MR imaging vs scintigraphy. Am J Roentgenol 1990, 55:1043-1048.

6. Molino A, Colombatti M, Bonetti F, Zarrdini M, Pasini F, Perini A, Pelosi G, Tridente G, Veneri D, Cetto GL: A comparative analysis of three different techniques for the detection of breast cancer cell in bone marrow. Cancer 1991, 67:1033-1036.

7. Mattano LA, Moss T, Emerson SG: Sensitive detection of rare circulating neuroblastoma cells by the reverse transcriptase-polymerase chain reaction. Cancer Res 1992, 52:47014705.

8. Campana $\mathrm{D}$, Pui $\mathrm{CH}$ : Detection of minimal residual disease in acute leukemias: methodological advances and clinical significance. Blood 1995, 85:1416-1434.

9. Keene SA, Demeure MJ: The clinical significance of micro metastases and molecular metastases. Surgery 2001, 129:15.

10. Gusteron B, Ott R: Occult axillary lymph-node micrometastases in breast cancer. Lancet 1990, 336:434-435.

11. Schoenfeld A, Luqmani $Y$, Smith D: Detection of breast cancer micro metastases in axillary lymph nodes using polymerase chain reaction. Cancer Res 1994, 54:2986-2990.

12. Nishiyama T, Sasaki T, Takaishi $K$, Kato M, Yaku H, Araki K, Matsuura $Y$, Takai $Y$ : rac p21 is involved in insulin-induced membrane ruffling and rho p21 is involved in hepatocyte growth factor- and 12-0-tetradecanoylphorbol-13-acetate (TPA)induced membrane ruffling in KB cells. Mol Cell Biol 1994, 14:2447-2456.

13. Ridley AJ, Comoglio PM, Hall A: Regulation of scatter factor/hepatocyte growth factor responses by Ras, Rac, and Rho in MDCK cells. Mol Cell Biol 1995, 15:1110-1122.

14. Jeffers M, Rong S, Woude GF: Hepatocyte growth factor/scatter factor-Met signaling in tumorigenicity and invasion/metastasis. J Mol Med 1996, 74:505-513.

15. Bladt F, Riethmacher D, Isenmann S, Aguzzi A, Birchmeier C: Essential role for the c-Met receptor in the migration of myogenic precursor cells into the limb bud. Nature 1995, 376:768771.

16. Schmidt C, Bladt F, Goedecke S, Brinkmann V, Zschiesche W, Sharpe M, Gherardi E, Birchmeier C: Scatter factor/hepatocyte growth factor is essential for liver development. Nature 1995, 373:699-702

17. Giordano S, Zhen Z, Medico E, Gaudino G, Galimi F, Comoglio PM: Transfer of motogenic and invasive response to scatter factor/hepatocyte growth factor by transfection of human MET protooncogene. Proc Natl Acad Sci USA 1993, 90:649-653.

18. Matsumoto K, Matsumoto K, Nakamura T, Kramer RH: Hepatocyte growth factor/scatter factor induces tyrosine phosphorylation of focal adhesion kinase (p125FAK) and promotes migration and invasion by oral squamous cell carcinoma cells. J Biol Chem 1994, 269:31807-31813.

19. Rong S, Bodescot M, Blair D, Dunn J, Nakamura T, Mizuno K, Park M, Chan A, Aaronson S, Vande Woude GF: Tumorigenicity of the met proto-oncogene and the gene for hepatocyte growth factor. Mol Cell Biol 1992, 12:5152-5158.

20. Rosen EM, Knesel J, Goldberg ID, Jin L, Bhargava M, Joseph A, Zitnik R, Wines J, Kelley M, Rockwell S: Scatter factor modulates the metastatic phenotype of the EMT6 mouse mammary tumor. Int J Cancer 1994, 57:706-714.

21. Leake R, Barnes D, Pinder S, Ellis $Y$, Anderson L, Anderson T, Adamson R, Rhodes T, Miller K, Walker R: Immunohistochemical detection of steroid receptors in breast cancer: a working protocol. J Clin Pathol 2000, 53:634-635.

22. Parr C, Jiang WG: Expression of hepatocyte growth factor/ scatter factor, its activator, inhibitors and the c-Met receptor in human cancer cells. Int J Oncol 2001, 19:857-863.

23. Tomioka $D$, Maharani $N$, Kuba $K$, Mizumoto $K$, Tanaka M, Matsumoto $\mathrm{K}$, Nakamura T: Inhibition of growth, invasion, and metastasis of human pancreatic carcinoma cells by NK4 in an orthotopic mouse model. Cancer Res 2001, 61:7518-7524.

24. Kermorgant S, Aparicio T, Dessirier V, Lewin MJ, Lehy T: Hepatocyte growth factor induces colonic cancer cell invasiveness via enhanced motility and protease overproduction. Evidence for PI3 kinase and PKC involvement. Carcinogenesis 2001, 22:1035-1042.

25. Wang Y, Selden AC, Morgan N, Stamp GWH, Hodgson HJF: Hepatocyte growth factor/scatter factor expression in human mammary epithelium. Am J Pathol 1994, 144:675-681.

26. Jin L, Fuchs A, Schnitt SJ, Yao Y, Joseph A, Lamszus K, Park M, Goldberg ID, Rosen EM: Expression of scatter factor and c-met receptor in benign and malignant breast tissue. Cancer 1997, 15:749-761.

27. Tuck AB, Park M, Sterns EE, Boag A, Elliott BE: Coexpression of hepatocyte growth factor and receptor met in human breast carcinoma. Am J Pathol 1996, 148:225-231.

28. Elliott $B E$, Hung $W L$, Boag $A H$, Tuck $A B$ : The role of hepatocyte growth factor (scatter factor) in epithelial mesencymal transition and breast cancer. Can J Physiol Pharmacol 2002, 80:91102.

29. Maemura M, Lino $Y$, Yokoe T, Horiguchi J, Takei H, Koibuchi $Y$, Horii Y, Takeyoshi I, Ohwada S, Morishita Y: Serum concentration of hepatocyte growth factor in patients with metastatic breast cancer. Cancer Lett 1998, 24:215-220.

30. Yamashita Jl, Ogawa M, Yamashita SI, Nomura K, Kuramoto M, Saishoji T, Shin S: Immunoreactive hepatocyte growth factor is a strong and independent predictor of recurrence and survival in human breast cancer. Cancer Res 1994, 54:1630-1633.

31. Shimabukuro K, Ichinose S, Koike R, Kubota T, Yamaguchi M, Miyasaka M, Aso T: Hepatocyte growth factor/scatter factor is implicated in the mode of stromal invasion of uterine squamous cervical cancer. Gynecol Oncol 2001, 83:205-215.

32. Tacchini L, Dansi P, Matteucci E, Desiderio MA: Hepatocyte growth factor signalling stimulates hypoxia inducible factor-1 (HIF-1) activity in HepG2 hepatoma cells. Carcinogenesis 2001, 22:1363-1371.

33. Kermorgant S, Dessirier V, Lewin MJ, Lehy T: HGF upregulates and modifies subcellular distribution of proteins in colon cancer cell enterocytic differentiation. Am J Physiol Gastrointest Liver Physio/ 2001, 281:G1068-G1080.

34. Marchetti A, Buttitta F, Bertacca G, Zavaglia K, Bevilacqua G, Angelucci D, Viacava P, Naccarato A, Bonadio A, Barassi F, Felicioni L, Salvatore S, Mucilli F: mRNA markers of breast cancer nodal metastases: compression between mammoglobin and carcinoembryonic antigen in 248 patients. J Pathol 2001, 195:186-190.

35. Yuan Rq RQ, Fan S, Achary M, Stewart DM, Goldberg ID, Rosen EM: Altered gene expression pattern in cultured human breast cancer cells treated with hepatocyte growth factor/scatter factor in the setting of DNA damage. Cancer Res 2001, 61:8022-8031.

36. Skibinski G, Skibinska A, James K: Hepatocyte growth factor (HGF) protects c-met-expressing Burkitt's lymphoma cell lines from apoptotic death induced by DNA damaging agents. Eur J Cancer 2001, 37:1562-1569.

\section{Correspondence}

Ron Greenberg MD, Department of Surgery A, Tel-Aviv Sourasky Medical Center, 6 Weizmann Street, Tel-Aviv 64239, Israel. Tel: +972 3 6973255; fax: +972 36974621 ; e-mail: rongree@mailcity.com 